\title{
Mechanism analysis of rain-wind induced vibration on high-voltage transmission line
}

\author{
Li Li, Chao Zhou and Yanping Liu \\ School of Energy Power and Mechanical Engineering \\ North China Electric Power University \\ Beijing China \\ lilizar@163.com
}

\begin{abstract}
Under rain-wind conditions, rain-wind induced vibration on high-voltage transmission line often occurs, this kind of vibration intensifies the fatigue of high-voltage conductors and tower collapse. The mechanism of rain-wind induced has aroused wind attention in power engineering. The presence of raindrops and wind associated with electric filed maybe the main cause of this phenomenon. In the conditions of rain-wind excitation, raindrops hit the transmission line. The suspended raindrops may be reformed as upper rivulet and lower rivulet. The rivulets are enlarged as a cone shape with the electric field of high-voltage conductor. The motion of upper rivulet and its effect on the original cross-section formation of the high-voltage conductor are likely to be the cause of rain-wind induced vibration. Based on the mechanism, a two-dimensional model of rain-wind vibration is established based on D'Alembert principle. Taking the conductor LGJ-240 as an instance, we investigate the effects of wind velocity, rivulet position, and electric field strength on the vibration amplitude. Mechanism and conclusions can be used as references for constructing high-voltage transmission, upgrading the existing lines and controlling vibration.
\end{abstract}

Keywords-rain-wind induced vibration; high-voltage transmission line; rivulet

\section{INTRODUCTION}

In the planning of ultra-high-voltage transmission lines that crossover southeast regions of china, one of the key questions are how to ensure secure and stable operation of the highvoltage transmission line under the conditions of a complex terrain, rainfalls, and the wind. Under the rainy weather, rainwind induced vibration on high-voltage transmission line often occurs. This kind of vibration can cause metal fatigue, especially on metal fitting and tower structure.

In recent years, many methods are taken to investigate the mechanism of rain-wind induced vibration. Kikuchi $[1,2]$ sets up a watering grid upwind in the wind-tunnel's test section to simulate aerodynamic characteristics of over-head power lines in heavy rainfall and wind. $\mathrm{Li}[3,4]$ takes rainfall as a shock load to combine with wind turbulence and proposes a calculation method for the combination of rain excitation with wind force. Zhou [5-7] investigates the effects of ionic wind, rainfalls, and nonlinear cross-sections on the vibration amplitude of the high-voltage transmission line. However, few works on the effects of rivulet motion, electric field strength,

National Science Foundation of China (No.51575180, 51205128) Beijing Natural Science Foundation (No.8152027) and wind velocity for the mechanism of this vibration are carried out. For this reason, the main objective of the present work is to reveal the mechanism by developing a validated two-dimensional model, which takes the variation of several factors such as wind velocity, rivulet position, and electric field strength into account.

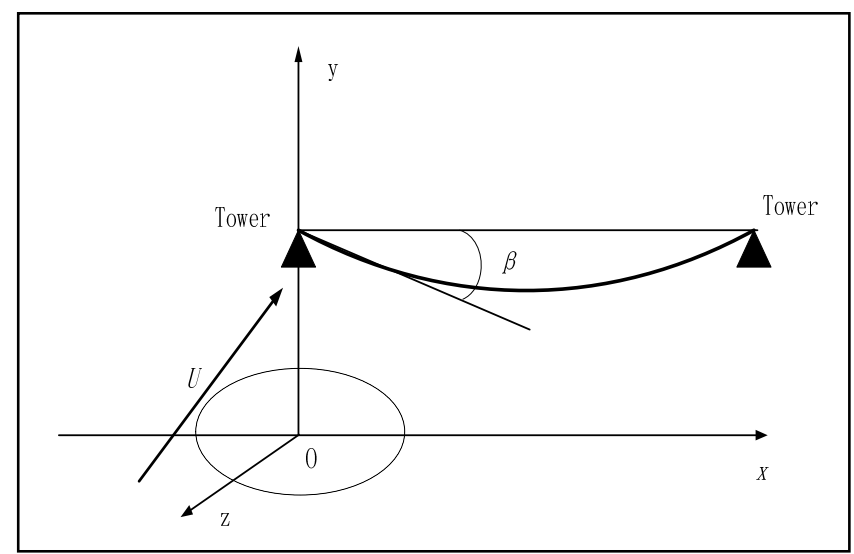

Fig. 1. . Sketch of the high-voltage conductor.

\section{TWO DIMENSIONAL MODEL AND VIBRATION EQUATION}

In order to facilitate analysis, we make some feasible assumptions to establish the two dimensional model as follow: the lower rivulet almost can be neglected, and ionic wind will not be taken into consideration, only considering the effect of electric field strength on the shape of the upper rivulet, and the quasi-steady state assumption is valid. Thus, let us use an elastic and uniformed cylinder with a small sag-to-span ratio to represent the high-voltage conductor (Fig. 1). The inclination of the cylinder is denoted by angle $\beta$, and $U$ denotes the mean wind velocity. Fig. 2 shows a two-dimensional model with relative wind velocity acting on the cross-section of cylinder and movement of the upper rivulet. The stable position of the upper rivulet is denoted by $\theta_{0}$, and $\theta$ denotes the vibration angle of the upper rivulet near the stable position. The uniform wind velocity is denoted by $U$, and the angel between the z-axis is $\gamma$. $U_{\text {rel }}$ denotes the relative wind velocity, and the angel between 
the z-axis is $\phi$. The drag coefficient $C_{D}$ is indicated in the direction of the resultant wind velocity $U_{r e l}$, whereas the lift coefficient $C_{L}$ is perpendicular to $C_{D}$ in anticlockwise direction. External forces acting on the cross-section of the transmission line are shown in Fig. $3 m_{0}$ and $C_{0}$ denote the mass of rivulet per unit length, damping of the rivulet. $f_{\theta}$ and $F e$ denote tangential and normal aerodynamic forces act on rivulet per unit length.

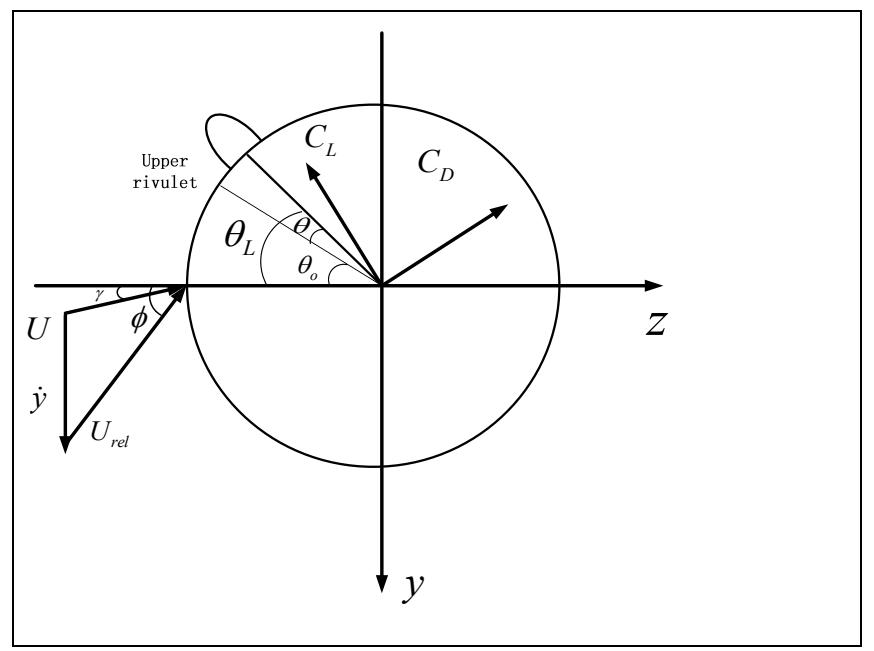

Fig. 2. Relations of upper rivulet and wind velocity.

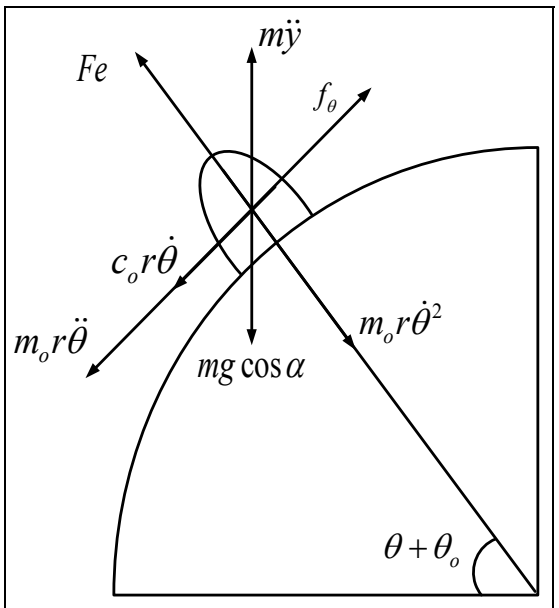

Fig. 3. External forces acting on the cross-section of the transmission.

Based on the above analysis, the two-dimensional 2-dof equation of rain-wind induced vibration of the conductor can be derived based on D'Alembert principle as follows:

$$
\begin{aligned}
& m \ddot{y}+c_{y} \dot{y}+k y+\left(f_{\theta}-m_{0} r \ddot{\theta}-c_{\theta} r \dot{\theta}\right) \cos \left(\theta+\theta_{0}\right)+ \\
& \left(F e-m_{0} r \dot{\theta}\right) \sin \left(\theta+\theta_{0}\right)+f_{y}(y, t)=0
\end{aligned}
$$

$$
m_{0} r \ddot{\theta}-(m \ddot{y}-m g \cos a) \cos \left(\theta+\theta_{0}\right)-f_{\theta}+c_{\theta} r \dot{\theta}=0
$$

Considering $\mathrm{m}_{0}<<\mathrm{m}$, simplify the above equation as follows:

$$
\begin{aligned}
& \ddot{y}+2 \xi_{y} \omega_{y} \dot{y}+\omega_{y}^{2} y=\frac{\left(c_{\theta} r \dot{\theta}-f_{\theta}\right) \cos \left(\theta+\theta_{0}\right)}{m} \ddot{\theta} \\
& -\frac{f_{y}(y, t)-F \sin \left(\theta+\theta_{0}\right)}{m} \ddot{\theta} \\
& +2 \xi_{\theta} \omega_{\theta} \dot{\theta}=\frac{(m \ddot{y}-m g \cos a) \cos \left(\theta+\theta_{0}\right)+f_{\theta}}{m_{0} r}
\end{aligned}
$$

Where $\omega_{y}$ and $\omega_{\theta}$ denote the natural frequency of transmission line and rivulet. $\xi_{y}$ and $\xi_{\theta}$ denote the damping ratio of the conductor and the upper rivulet.

The equation of aerodynamic can be expressed as

$$
\begin{gathered}
f_{y}=\frac{1}{2} \rho D U_{r e l}^{2}\left(C_{L} \cos \phi+C_{D} \sin \phi\right) \\
f_{\theta}=\frac{1}{2} \rho r^{\prime} D U_{r e l}^{2}\left[\begin{array}{l}
C_{l} \cos \left(\phi+\theta+\theta_{0}\right)+ \\
C_{d} \cos \left(\phi+\theta+\theta_{0}\right)
\end{array}\right]
\end{gathered}
$$

Where $\rho, D$ and $r$ denote the density of air, diameter of conductor and equivalent radius of the rivulet. $C_{L}$ and $C_{D}$ denote aerodynamic lift coefficient and drag coefficient of the conductor. $C_{l}$ and $C_{d}$ [8] denote aerodynamic lift coefficient and drag coefficient of the rivulet, respectively.

\section{NUMERICAL ANALYSIS AND DISCUSSION}

To investigate the capability of the analytical model for revealing the rain-wind inducing vibration mechanism, the model is solved by Galerkin weighted residual method and central difference method. Taking the conductor LGJ-240 as an instance, the key parameters of the high-voltage transmission line are taken as diameter of $20 \mathrm{~mm}$, mass per unit length of $0.96 \mathrm{~kg} / \mathrm{m}$, elasticity coefficient of $73 \mathrm{KN} / \mathrm{mm}^{2}$, tension force of $8.31 \mathrm{KN}$, the damping ratio of 0.2 , the natural frequency of 6 $\mathrm{rad} / \mathrm{s}$ and mixed density of air and rain can be derived as 1.5 $\mathrm{kg} / \mathrm{m}^{3}$, with a wind velocity of $8 \mathrm{~m} / \mathrm{s}$. Moreover, the damping ratio of rivulet is 0.03 .

\section{A. Effect of wind velocity on rain-wind induced vibration}

According to solving above equations based on central difference method, we can obtain the amplitude of vibration of the conductor, at $\beta=35^{\circ}, \alpha=30^{\circ}, \varphi=66^{\circ}, \theta_{0}=\pi / 2+\Phi-\varphi=0.76$, varies differently with different wind velocity (Fig. 4). Where $y$ denotes vibration curve of the displacement, and $d y$ denotes vibration curve of the velocity. The vibration amplitude of the high-voltage conductor increases almost linearly with the increasing of wind velocity up to $13 \mathrm{~m} / \mathrm{s}$. this phenomenon somehow likes galloping vibration, but, in fact it is found that the response amplitude is actually limited by the velocity $\dot{y}$ and the angle $\theta_{0}$. The results can be explained that the highvoltage conductor with fixed upper rivulet presents a velocityrestricted vibration response. 


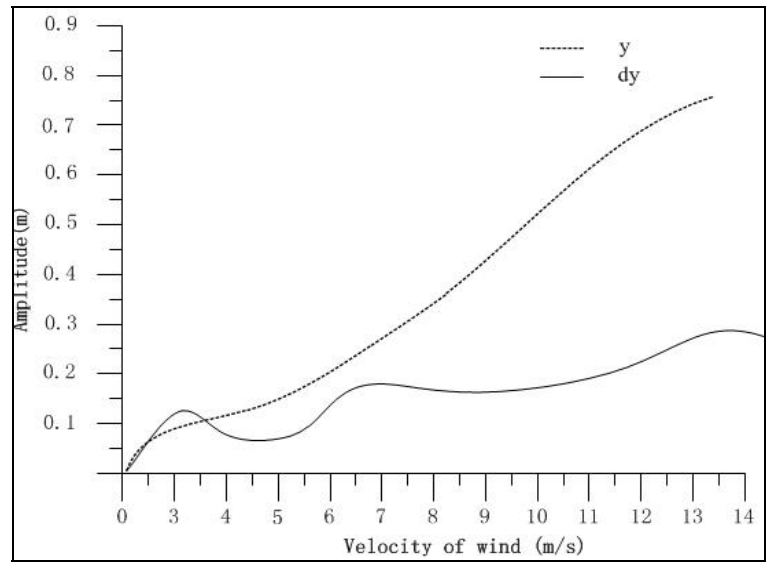

Fig. 4. The amplitude of rain-wind vibration with wind velocity.

\section{B. Effect of electric field strength on amplitude of transmission}

When the high-voltage transmission line is electrified, there is electric field around the surface of the conductor. Thus, water molecules are polarized, and the electric field strength $\mathrm{Fe}$ can be expressed as:

$$
F e=\frac{\pi \varepsilon_{0}}{4}\left[\frac{3\left(\varepsilon_{2}-\varepsilon_{1}\right)}{\varepsilon_{2}+2 \varepsilon_{1}}\right]^{2} E_{0}^{2} r^{2} \sin (\omega t)
$$

Effect of electric field strength on the amplitude of transmission is shown in Fig. 5, at $f=50 \mathrm{~Hz}, \omega=100 \pi$. It can easily find that the amplitude increases with the increasing of the electric field, when $V=0 \sim 500 K V$. Conversely, when $V=500 \sim 1000 \mathrm{KV}$, the amplitude decreases with the decreasing of the electric field. The result can be explained that the amplitude of the vibration is unstable when the electric field at $500 \mathrm{kV}$.

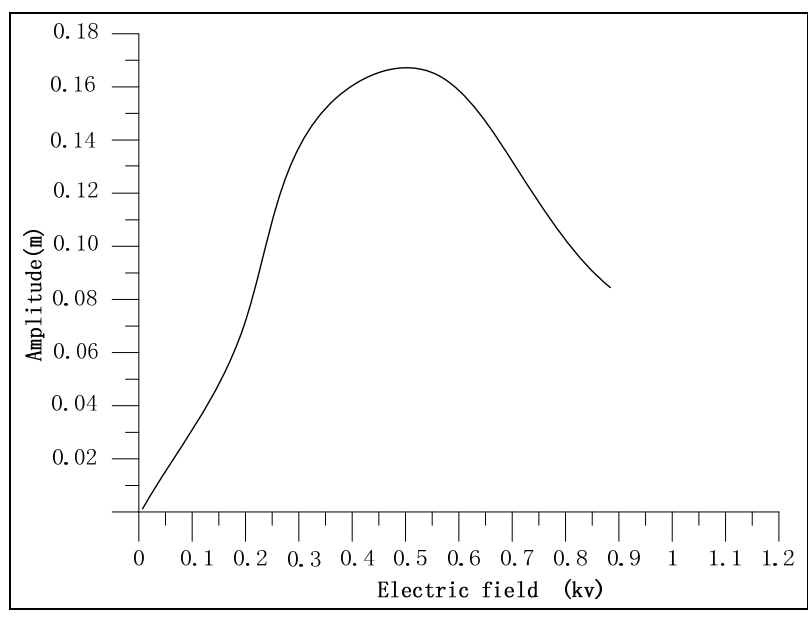

Fig. 5. The amplitude of rain-wind vibration with electric filed strength.

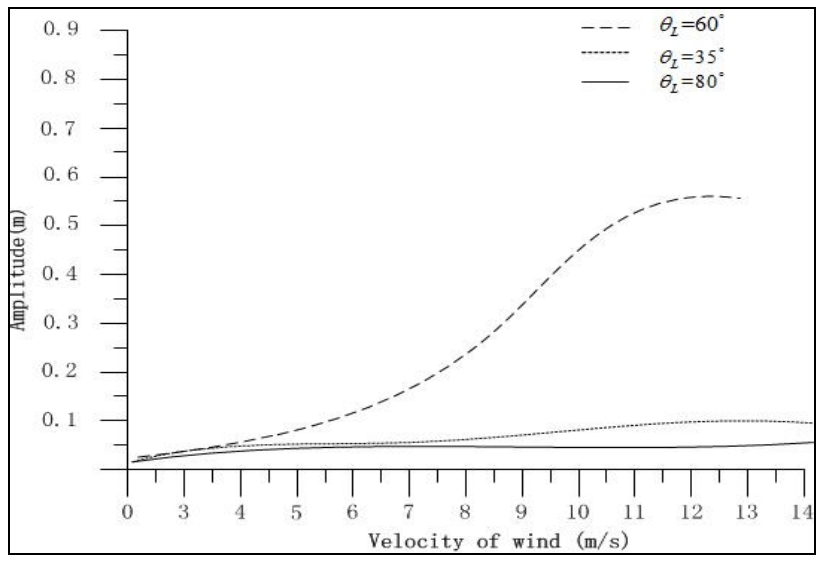

Fig. 6. Effect of the position of the rivulet with the wind on the rain-wind vibration.

\section{Effect of the position of the upper rivulet on rain-wind induced vibration}

For the aims of revealing the effect of the position of the rivulet on rain-wind induced vibration, we assume that the mass and the velocity is zero respectively and don't consider the impact of corona force. The coefficients $C_{D}, C_{L}$ can obtain from experimental data [9]. The values of interpolated in the range of interest, $1.048 \leq \varphi \leq 1.919\left(\varphi=\alpha+\theta_{L}\right)[10]$ and the coefficients $C_{D}, C_{L}$ can be functioned as:

$$
\left\{\begin{array}{l}
C_{L}(\varphi)=77.11 \varphi^{3}-366.18 \varphi^{2}+562.73 \varphi-276.12 \\
C_{D}(\varphi)=10.16 \varphi^{3}-48.06 \varphi^{2}+73.56 \varphi-35.83
\end{array}\right.
$$

Fig. 6 shows the maximum displacement of the transmission line, at the rivulet position of $\theta_{L}=35^{\circ}, 60^{\circ}, 80^{\circ}$, varies with the range of velocity wind from 0 to $13 \mathrm{~m} / \mathrm{s}$. From the comparison of these curves, it can easily find that the displacement increases with the increasing of the wind velocity when $\theta_{L}=60^{\circ}$. Conversely, once $C_{D}$ doesn't within the maxim sudden fall region, the transmission line essentially no vibration occurs. The result can be explained by the fact that at the same wind velocity, the transmission line activities in the lower region of the lift coefficient are usually stronger than the other of the lift coefficient.

\section{CONCLUSIONS}

For the aims of the revealing mechanism of rain-wind induced vibration of high-voltage transmission lines, the twodimensional 2-dof equation of rain-wind induced vibration model presented is presented in this paper, the model based on D'Alembert principle and central difference method. Take the conductor LGJ-240 as an instance, we investigate the effects of wind velocity, rivulet position, and electric filed strength on the amplitude of the conductors. The result shows wind velocity, rivulet position, and electric filed strength have obvious effects on rain-wind induced vibration. Mechanism and conclusions in this paper can be used as references to construct high-voltage 
transmission lines, upgrade the existing lines and control vibration.

\section{REFERENCES}

[1] N. Kikuchi, Y. Matsuzaki, T. Yukino, and H. Ishida, "Aerodynamic drag of new-design electric power wire in a heavy rainfall and wind," J. Wind Eng. Ind. Aerodynam. vol. 91, no.1-2, pp.41-51, 2003.

[2] Y. Eguchi, N. Kikuchi, K. Kawabata, T. Yukino, and Y. Ishikubo, "Drag reduction mechanism and aerodynamic characteristics of a newly developed overhead electric wire," J. Wind Eng. Ind. Aerodynam, vol. 90, no. 4, pp. 293-304, 2002.

[3] H.N. Li, Y.M. Ren, and H.F. Bai, "Rain-wind-induced dynamic model for transmission tower system," Proc. CSEE, vol. 27, no. 30, pp. 43-47, 2007.

[4] H.F. Bai, and H.N. Li. "Dynamic response of overhead transmission lines to oscillation caused by wind or rainfall loads," Power Syst. Technol. vol. 33, no. 2, pp. 36-40, 2009.

[5] C. Zhou, Y.B. Liu and X.M. Rui, "Mechanism and characteristic of raininduced vibration on the high-voltage transmission line," J. Mech. Sci. Technol. vol. 40, no. 8, pp. 2505-2510, 2012.

[6] C. Zhou, and Y.P. Liu, "Numerical analysis of rain-wind induced vibration on the conductor by finite element method," Appl. Mech. Mater. vol. 105, pp. 151-154, 2012.

[7] C. Zhou, and X.M. Rui, "Model of rain-induced vibration of the transmission line and numerical analysis." J. Vib. Shock, vol. 32, no. 6, pp. 173-175, 2013.

[8] M. Gu and X.Q. Du, "Quasi 2-DOF Model for Rain Wind Induced Vibration of 3-D Cables of Cable Stayed Bridges," Chinese Q. Mech. vol. 25, no. 4, pp. 496-501, 2004.

[9] Y.L Guo, G.X. Li, and C.Y. Long, "Dancing of the transmission line." China electric power press, Beijing, China, pp. 236-242, 2003.
[10] T. Li, Z.Q. Chen and S.Y. Li, "Modes coupling and in plane/out-ofplane in rain-wind induced vibration of cable," J. Vib. Shock, vol. 31, no. 4, pp. 115-122, 2012. 